\title{
Metabolic glycan labeling-based screen to identify bacterial glycosylation genes
}

\author{
Karen D. Moulton, ${ }^{1}$ Adedunmola P. Adewale, ${ }^{1}$ Hallie A. Carol, ${ }^{1}$ Sage A. Mikami, ${ }^{1}$ and \\ Danielle H. Dube ${ }^{1 *}$ \\ ${ }^{1}$ Department of Chemistry \& Biochemistry, Bowdoin College, \\ 6600 College Station, Brunswick, Maine 04011
}

\section{Supporting Information}

Table of Contents

Supplemental Methods...........................................................................

Quantification of LPS in enriched and depleted samples...........................................

Viability assessment of wildtype and mutant $H$. pylori...........................................

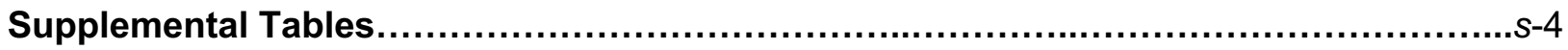

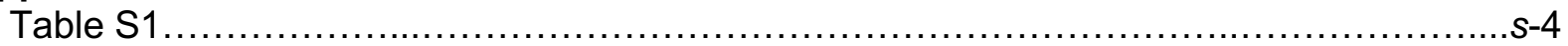

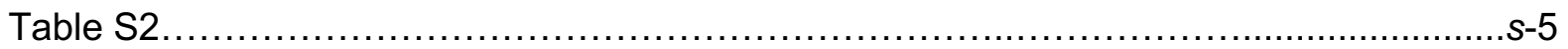

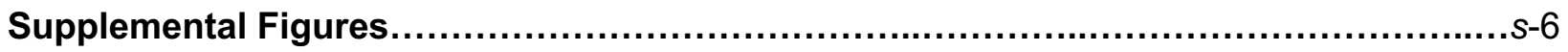

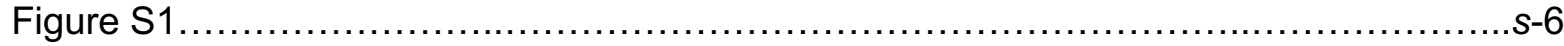

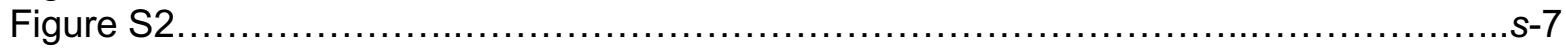

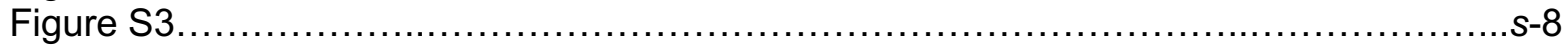

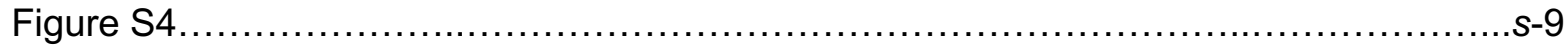

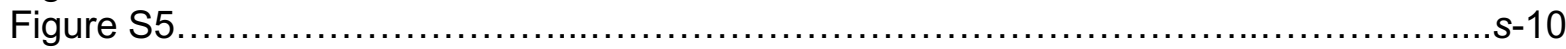

Supplemental References................................................................ 


\section{Supplemental Methods}

\section{Quantification of LPS in enriched and depleted samples}

The ToxinSensor ${ }^{\mathrm{TM}}$ Chromogenic LAL Endotoxin Assay Kit (GenScript) was used to detect the presence and concentration of LPS in a metabolically-labeled $H$. pylori whole cell lysate control, as well as in LPS-depleted and LPS-enriched samples. Limulus Amebocyte Lysate (LAL) was used in this assay according to manufacturer's instructions to detect the presence and concentration of LPS. A standard curve was constructed using E. coli standards at $0.125,0.25,1$, and 2 endotoxin units per $\mathrm{mL}(\mathrm{EU} / \mathrm{mL})$, provided by the kit. The concentration of LPS in each sample $(E U / m L)$ was calculated using the equation provided by the standard curve. Final LPS concentrations were calculated for the undiluted samples and converted to $\mathrm{ng} / \mathrm{mL}$. Note that in this assay $0.1 \mathrm{EU} / \mathrm{mL}$ approximates to $0.01 \mathrm{ng} / \mathrm{mL}$ of LPS.

\section{Viability assessment of wildtype and mutant $\mathrm{H}$. pylori}

Viability of mutant and wildtype $H$. pylori was assessed over the course of 4 days by enumerating colony forming units (CFUs) or by scoring percentage of live cells. $H$. pylori were inoculated at a starting optical density at $600 \mathrm{~nm}\left(\mathrm{OD}_{600}\right)$ of $\sim 0.1-0.2$ into culture tubes containing $3 \mathrm{~mL}$ of Brucella Broth. To measure CFUs, cells were harvested at 0 or 4 days, serially diluted, and grown on horse blood agar plates in duplicate under microaerophilic conditions. The number of colony forming units were enumerated after five days of incubation, once colonies became visible and could be counted. In parallel to scoring CFUs, percentage of live cells in cultures was measured using the LIVE/DEAD BacLight Bacterial Viability and Counting Kit (Invitrogen) according to manufacturer's instructions. Briefly, cells were rinsed with $0.85 \% \mathrm{NaCl}$ three times before staining with LIVE/DEAD BacLight Bacterial Viability solution, consisting of propidium iodide and SYTO 9 diluted in $0.85 \% \mathrm{NaCl}$ according to manufacturer's instructions. The cells were kept in the dark for 15 min and then analyzed by flow cytometry using a BD Accuri C6 Plus (BD Biosciences) instrument, with 20,000 live cells gated for each 
replicate experiment. Cells were scored as live or dead by using gates established with live and dead controls. The number of dead (red) and live (green) $H$. pylori cells were counted using FlowJo software (Ashland, OR) to determine the percentage of live H. pylori (percentage live cells $=100^{*}[(\#$ live cells $) /(\#$ live cells $+\#$ dead cells $\left.)]\right)$. 


\section{Supplemental Tables}

Table S1. List of primers used to amplify target genes to assess proper mutant strain construction.

\begin{tabular}{|c|c|}
\hline Primer name & Sequence (5' to 3') $^{\prime}$ \\
\hline HpG27_94 forward & gtcatatgttaaaagtttctgtgatcacg \\
\hline HpG27_94 reverse & catctagattaatcgtttcgtttaatccc \\
\hline HpG27_190 forward & acatatggtggaaaatttaagcgc \\
\hline HpG27_190 reverse & ctctagattaaaaaacccttttaatcaatcg \\
\hline HpG27_579 forward & acatatgattggtgtctatatcatttctttaaa \\
\hline HpG27_579 reverse & ctctagatcaataatagagtcttgttgcg \\
\hline HpG27_580 forward & acatatggttttagaaaaattaaaagaaaactc \\
\hline HpG27_580 reverse & ctctagattaaacctctttaggggttttaa \\
\hline HpG27_607 forward & acatatgcgttttttaccttgttttt \\
\hline HpG27_607 reverse & ctctagatcatggtttgtcctttgag \\
\hline HpG27_613 forward & acatatgttccaaccctattagacg \\
\hline HpG27_613 reverse & attctagattatttttaacccatctccttatg \\
\hline HpG27_645 forward & acatatgttgtgcgtgtttgatatagaa \\
\hline HpG27_645 reverse & ctctagatcatggtttgtcctttgag \\
\hline HpG27_761 forward & ccatatgcgtgttttattattcatttaag \\
\hline HpG27_761 reverse & agtctagactaatgttgccaattctgat \\
\hline HpG27_785 forward & acatatgcgtgttttatcatttcttaa \\
\hline HpG27_785 reverse & agtctagactatacaaactgccaatatttca \\
\hline HpG27_952 forward & gtggatccaattgaatcaagaagcgctcattaaagc \\
\hline HpG27_952 reverse & cactcgagttacgataaggttttaaagagatggg \\
\hline HpG27_1230 forward & acatatgatgacgcaacgcc \\
\hline HpG27_1230 reverse & agtctagatcacacttcttctatgaagtaatctt \\
\hline HpG27_1236 forward & gaggatccattttacccaactctctttatggcac \\
\hline HpG27_1236 reverse & cactcgagctacatcttgcggtaattgataaaacct \\
\hline HpG27_1339 forward & aacatatggatacgcaaaacttaccc \\
\hline HpG27_1339 reverse & agtctagattaagagagtttgaaaactttttca \\
\hline HpG27_1509 forward & tccatatgatgaataaagaagaagaatggaaaaagttac \\
\hline HpG27_1509 reverse & gctctagaaactttcatgcttgtaagacaaattc \\
\hline HpG27_1518 forward & gacatatgatgctatatttttaaccagtttatttatttgctcttg \\
\hline HpG27_1518 reverse & gctctagatcattgaaacgctcgcttttctaaagcataaacttgcc \\
\hline
\end{tabular}


Table S2. List of strains used in this study.

\begin{tabular}{|c|c|c|}
\hline Strain name & Genotype & Notes \\
\hline H. pylori G27 & wildtype & sequenced $^{[1]}$ \\
\hline$\Delta 94$ & $94::$ cat & transposon location $107852^{[2]}$ \\
\hline$\Delta 146$ & $146::$ cat & transposon location $163927^{[2]}$ \\
\hline$\Delta 190$ & 190::cat & transposon location $210477^{[2]}$ \\
\hline$\Delta 579$ & 579::cat & targeted insertion $^{[3]}$ \\
\hline$\Delta 580$ & 580::cat & targeted insertion $^{[3]}$ \\
\hline$\Delta 607$ & $607::$ cat & targeted insertion ${ }^{[3]}$ \\
\hline$\Delta 613$ & 613::cat & targeted insertion $^{[3]}$ \\
\hline$\Delta 645$ & 645:cat & transposon location $706310^{[2]}$ \\
\hline$\Delta 761$ & $761::$ cat & targeted insertion $^{[3]}$ \\
\hline$\Delta 785$ & $785:$ :cat & transposon location $849799^{[2]}$ \\
\hline$\Delta 952$ & 952::cat & targeted insertion $^{[3]}$ \\
\hline$\Delta 1018$ & 1018::cat & targeted insertion ${ }^{[3]}$ \\
\hline$\Delta 1036$ & 1036::cat & transposon location $1140370^{[2]}$ \\
\hline$\Delta 1046$ & 1046::cat & targeted insertion $^{[3]}$ \\
\hline$\Delta 1230$ & 1230::cat & transposon location $1349080^{[2]}$ \\
\hline$\Delta 1235$ & 1235::cat & transposon location $1353098^{[2]}$ \\
\hline$\Delta 1236$ & 1236::cat & transposon location $1354124^{[2]}$ \\
\hline$\Delta 1339$ & 1339::cat & transposon location $1450987^{[2]}$ \\
\hline$\Delta 1509$ & 1509::cat & targeted insertion $^{[3]}$ \\
\hline$\Delta 1515$ & 1515::cat & targeted insertion $^{[3]}$ \\
\hline$\Delta 1518$ & 1518::cat & targeted insertion $^{[3]}$ \\
\hline
\end{tabular}




\section{$\underline{\text { Supplemental Figures }}$}
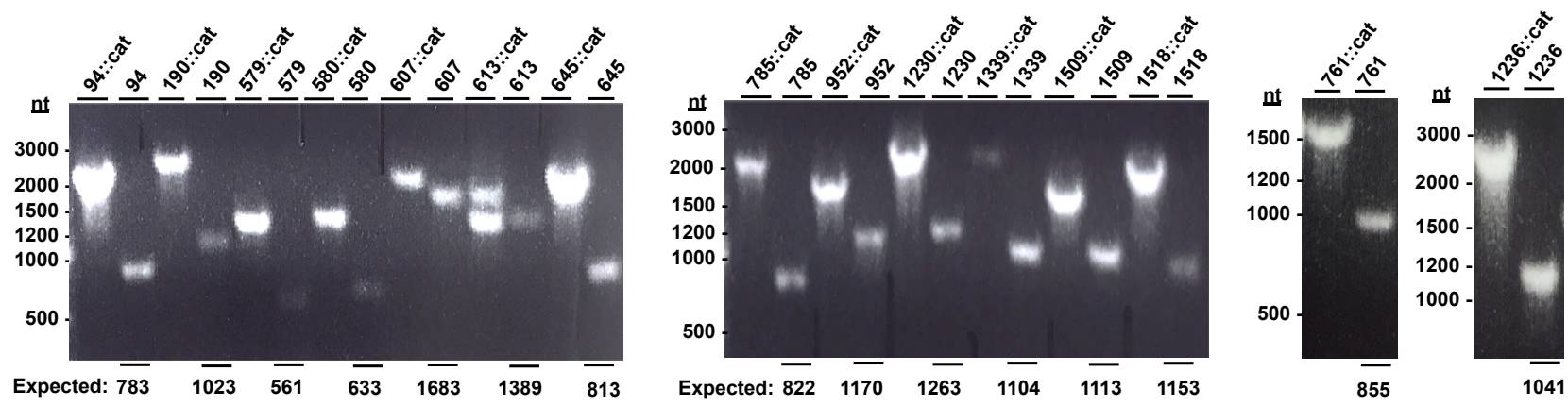

Figure S1. PCR amplification of genes insertionally inactivated with a chloramphenicol acetyltransferase cassette confirms successful construction of mutant strains. Primers specific to the 5' and 3' end of target genes were used to amplify each gene using genomic DNA from wild type $H$. pylori G27 or from the indicated mutant strain (e.g. 94::cat) as template. In all mutant strains, a high molecular weight band consistent with insertion of the chloramphenicol acetyltransferase (cat) cassette $\left(\mathrm{CAM}^{\mathrm{R}}\right)$ into the target gene is observed. In all controls, amplification of target genes from wild type genomic DNA yielded a low molecular weight band consistent with the expected molecular weight of each gene. The expected molecular weight of each wild type gene (e.g. HpG27_94 is $783 \mathrm{nt}$ ) is indicated below the lane of the gel in which that gene was amplified. 
A) Glycoprotein biosynthesis fingerprints of mutant strains

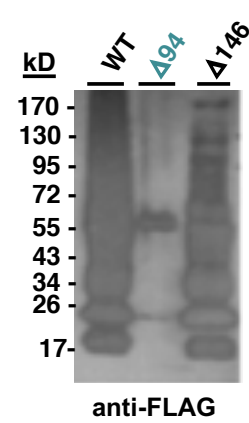

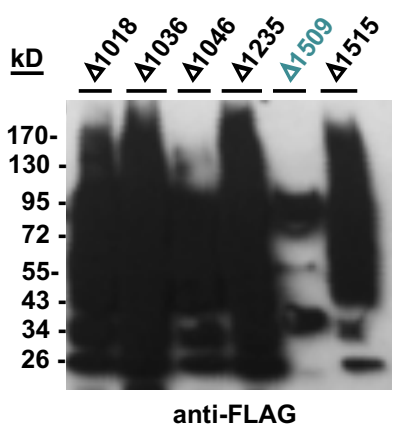

B) Corresponding protein loading from samples in (A)

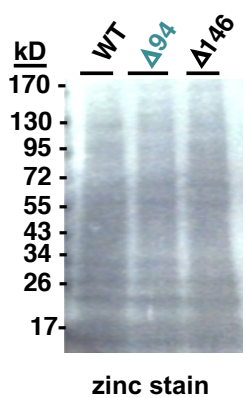

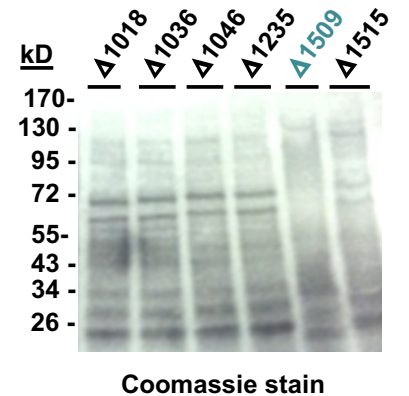

Coomassie stain

C) MOE benchmarks

D) Protein loading of samples from anti-FLAG probing in Figure 2B

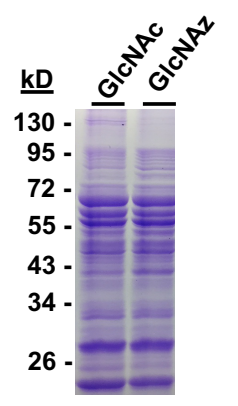

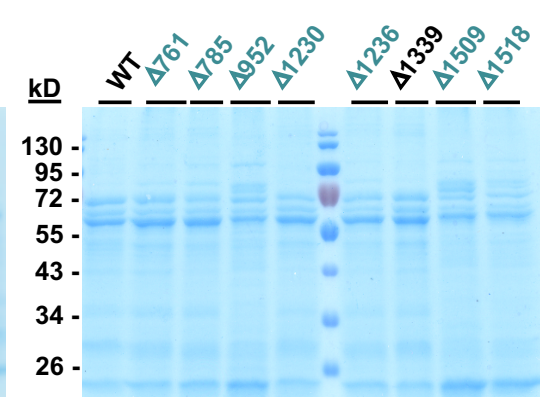

E) Protein loading from O-linked glycan removal

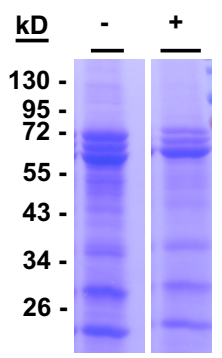

Figure S2. Metabolic glycan labeling screen of additional mutant strains, and protein loading for samples presented in Figure 2. A) Wild type (WT) and mutant G27 strains bearing insertionally inactivated glycosylation genes strains were screened for their ability to biosynthesize the full profile of azide-labeled glycoproteins upon treatment with the azidecontaining sugar $\mathrm{Ac}_{4} \mathrm{GlcNAz}$ for four days. Following metabolic labeling, samples were lysed, reacted with Phos-FLAG, and analyzed via western blot with anti-FLAG antibody. B) Coomassie or zinc staining of electrophoresed samples from panel (A) revealed that all western samples contain roughly equivalent protein levels. C, D, E) Coomassie staining of electrophoresed samples from Figure 2 revealed that all western samples contain roughly equivalent protein levels. 


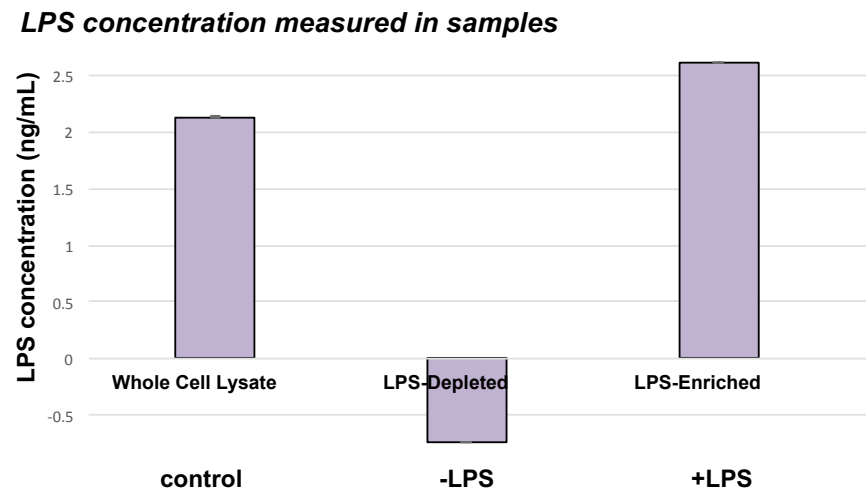

Figure S3. LPS quantification assay indicates successful LPS depletion and LPS enriched from LPS-depleted and LPS-enriched samples, respectively. LPS-depleted and LPS-enriched samples were quantified using the ToxinSensor ${ }^{\mathrm{TM}}$ LPS detection assay (GenScript) to determine the concentration of LPS in various H. pylori samples. The concentration of LPS in the azide-labeled LPS-depleted sample was below the detection limits of this assay $(0.05 \mathrm{ng} / \mathrm{mL})$. Error bars represent the standard deviation of three replicates. 
Proteinase K treatment ablates azide but not Ley signal
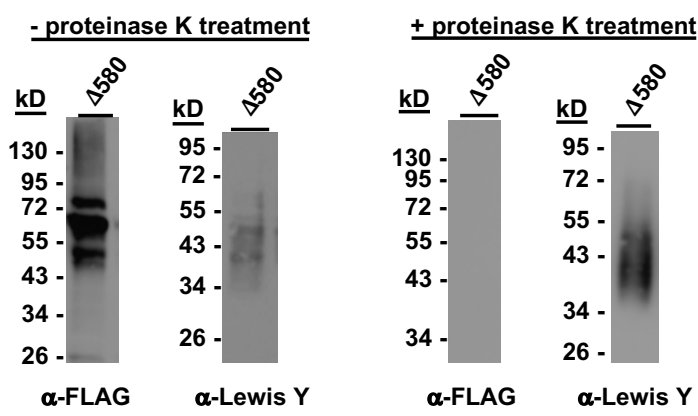

Figure S4. Azides are present in $H$. pylori proteins, whereas the Lewis $Y$ antigen is present in both proteins and LPS. Lysates from metabolically-labeled mutant strain $\Delta 580$ were probed for the presence of azides and Lewis $\mathrm{Y}$ antigen before (-) or after (+) proteinase $\mathrm{K}$ treatment. Proteinase $\mathrm{K}$ treatment ablated azide-dependent signal but not Lewis $\mathrm{Y}$-dependent signal, suggesting that azides are present in glycoproteins but not in LPS. 
A) H. pylori viability, scored by colony forming units*

$\begin{array}{cccc}\text { Day } & \frac{\mathbf{W T}}{64} & \frac{\Delta 579}{111} & \frac{\Delta 580}{46} \\ 0 & 206 & 128 & 218\end{array}$

* Tabulated values are CFUs x 106/mL
B) H. pylori viability, scored by LIVE/DEAD assay*

$\begin{array}{ccrr}\text { Day } & \underline{\mathbf{W T}} & \underline{\Delta 579} & \frac{\Delta 580}{99.5 \%} \\ 0 & 99.4 \% & 99.9 \% & 99.5 \% \\ 4 & 100 \% & 99.0 \% & 98.0 \% \\ \text { * Tabulated values are \% live cells }\end{array}$

Figure S5. Viability of wild type and mutant G27 H. pylori strains. A, B) H. pylori strains were cultured in liquid media and scored for viability by $(A)$ enumerating colony forming units (CFUs) and (B) measuring percent of live cells in each sample at 0 and 4 days, as noted. Data are representative of biological replicates. 


\section{Supplemental References}

[1] D. A. Baltrus, M. R. Amieva, A. Covacci, T. M. Lowe, D. S. Merrell, K. M. Ottemann, M. Stein, N. R. Salama, K. Guillemin, Journal of Bacteriology 2009, 191, 447-448.

[2] N. R. Salama, B. Shepherd, S. Falkow, Journal of Bacteriology 2004, 186, 7926-7935.

[3] D. C. Yang, K. M. Blair, J. A. Taylor, T. W. Petersen, T. Sessler, C. M. Tull, C. K. Leverich, A. L. Collar, T. J. Wyckoff, J. Biboy, W. Vollmer, N. R. Salama, Journal of Bacteriology 2019, 201. 